\title{
Comparison of echocardiography, magnetic resonance imaging and histopathology for the imaging evaluation of intracardiac masess
}

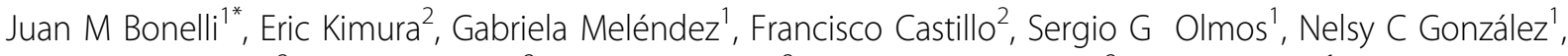
Erick Alexánderson ${ }^{3}$, Luis Marroquin ${ }^{3}$, Fernando Iñarra ${ }^{3}$, José E Telich-Tarriba ${ }^{3}$, Aloha Meave ${ }^{1}$

From 15th Annual SCMR Scientific Sessions

Orlando, FL, USA. 2-5 February 2012

\section{Background}

Cardiac Magnetic Resonance (CMR) has demonstrated being an ideal method in the diagnosis of cardiac masses because of its accuracy in tissue characterization. The main objective was to compare the utility of CMR in the diagnosis of cardiac masses with Echocardiography (Echo) having the histopathology findings as the gold standard.

\section{Methods}

Thirty four patients were enrolled: twenty two males, twelve females, age between 15 days and 80 years old (mean 38 years old) with diagnosis of cardiac mass underwent echo and CMR before biopsy or surgery. Echo gradient cine images in multiple views, $\mathrm{T} 1$ and $\mathrm{T} 2$ weighted sequences, and additional information derived from first pass perfusion imaging and inversion recovery post gadolinium delayed images allowed an accurate diagnosis in the majority of cases.

\section{Results}

Table 1 summarizes the main results. MRI was able to diagnose correctly 26 of 34 cases $(76.5 \%)$ while echo only diagnosed correctly 11 of 34 cases $(32.3 \%)$.

\section{Conclusions}

We conclude that CMR is an advantageous tool over echo in detection, and complete morphological and functional evaluation as hemodynamic repercussion of cardiac masses. This research also demonstrated CMR capacity to exclude or confirm the presence of a cardiac mass when the echo was equivocal. CMR predicts the likely diagnosis of the tumor in the majority of the cases. A comprehensive imaging protocol is essential for accurate diagnosis. However, histopathology diagnosis remains the gold standard, and in some cases malignancy cannot be definitively excluded on the basis of CMR images alone.

\section{Funding}

National Institute of Cardiology "Ignacio Chávez", Mexico City, Mexico.

\section{Author details}

${ }^{1}$ Cardiovascular Magnetic Resonance Imaging, National Institute of Cardiology "Ignacio Chávez", Mexico City, Mexico. ${ }^{2}$ Cardiovascular Computed Tomography, National Institute of Cardiology "Ignacio Chávez", Mexico City, Mexico. ${ }^{3}$ Nuclear Cardiology, National Institute of Cardiology "Ignacio Chávez", Mexico City, Mexico.

Published: 1 February 2012

doi:10.1186/1532-429X-14-S1-P295

Cite this article as: Bonelli et al:: Comparison of echocardiography, magnetic resonance imaging and histopathology for the imaging evaluation of intracardiac masess. Journal of Cardiovascular Magnetic Resonance 2012 14(Suppl 1):P295. 
Table 1 Results

\begin{tabular}{cccc}
\hline Masses & Echocardiogram diagnosis & Cardiovascular MRI diagnosis & Histopathology diagnosis \\
\hline Thrombus & $4(2 / 8)$ & $7(7 / 8)$ & 10 \\
Myxoma & $8(7 / 10)$ & $9(9 / 10)$ & 10 \\
Sarcoma & - & $5(2 / 2)$ & 2 \\
Endomyocardial fibrosis & - & $3(2 / 2)$ & 2 \\
Rhabdomyoma & $3(1 / 2)$ & $3(2 / 2)$ & 2 \\
Pericardial mass & 2 & 2 & - \\
Other & $1(1 / 5)$ & $3(2 / 5)$ & 5 \\
Papillary fibroelastoma & - & $1(1 / 1)$ & 1 \\
Metastasis & - & $1(1 / 2)$ & 2 \\
Non-specific & 16 & - & - \\
Total & - & - & 2 \\
\hline
\end{tabular}

